\title{
NOVEL INTERACTIVE HUMAN-ROBOT-SYSTEMS
}

\author{
D. Šurdilović and R. Bernhardt
}

Fraunhofer IPK, Berlin, Germany

\begin{abstract}
This paper presents several novel interactive human-robot systems which were recently developed at IPK. These systems were designed for direct physical interaction between humans and robots. The principal structures and functions of the following robotic systems are briefly presented: ROBOPED, an advanced parallel robot for orthopedic diagnostics and rehabilitation, STRING-MAN, a wire robot for gait rehabilitation, and IPK's Kobot, a collaborative manipulator for advanced material handling and assembly. Copyright @ 2005 IFAC
\end{abstract}

Keywords: robot control, rehabilitation, manipulators, cobots

\section{INTRODUCTION}

Robots were created in order to automate, optimize ameliorate work processes that have up to now been carried out by humans alone. For reasons of security, humans may not enter the working space of the robot. Recently it has been shown, however, that robots cannot come close to matching the abilities or intelligence of humans. Therefore, new systems which enable the interaction between humans and robots are becoming more and more important. The advantages of using innovative robots can especially be seen in medical or other service-oriented areas as well as in the emerging area of humanoid robots.

The interaction, i.e., the cooperation between human and robot, should be considered in its different aspects. A detailed Taxonomy of Human-Robot interaction (Yanko et al, 2002 and 2004) distinguishes between different categories of interaction according to various criteria: first, spatial- and time-based usage of computer resources by humans and robots (e.g., common, separate, in the same or different workspace), second, decision-level and autonomy (e.g. from autonomous operation to telemanipulation), and finally, the human role in the interaction (supervisor, operator, programmer, "working colleague," observer, etc.). A separate role is played by the physical distance, upon which the following action-categories are based: escape, approximate, go behind, outpace and touch.
Of the special interest, however still seldom put into practice, are applications requiring direct physical contact between robots and humans (or the environment in general). These tasks are refereed to as contact tasks or compliant tasks. Two basic groups within the contact tasks may be distinguished (Yanko et al, 2004). The first one involves the essential contact tasks, which explicitly require that the robot act upon the environment with a specific amount of force.

In the second sub-group of contact tasks, the main demands are made on the end-effector movements in the immediate vicinity of the working objects (constrained or compliance-movements). In the human-robot contact task, the human takes on the role of the environment and can act either passively (e.g., for robot applications in surgery) or actively (he can also exert force and on the robot). In an even more complex task, robots and humans can interact together with an additional environment (e.g., a work object).

The main problem to be dealt with in performing contact tasks is to safeguard against injury to the environment, or, even more important, to human beings. Robot strength presents a very serious potential danger to objects or people in the immediate area in case of mistakes. To prevent this and, at the same time, guarantee stable interaction, an appropriate strategy for a passive control of the robot must be deployed (Colgate et al, 1992). This can be done, on the one hand, via monitoring and controlling 
the contact forces, and on the other, by introducing compliant robot behavior. A classification of the various control strategies, that have been researched and experimentally tested as well as an overview of the control algorithms that have been developed are presented in (Vukobratovic and Surdilovic, 2002). Among the basic control techniques are: the force control, or, more generally described, the hybrid position/force control (mainly referring to the essential control tasks), and the impedance control (suitable primarily for flexible behavior).

The interactive human-robot systems which have recently been developed at Fraunhofer IPK will be described below. What each of these systems has in common are physical contact, as well as, interaction between robot and human. In the case of the rehabilitation robot, "Stringman," the human is a part of the robot system (he serves as a common platform within a wire robot). In addition, force and impedance control is used in each of the systems. A robust method for the control design was used, which therefore guarantees robust stability in the interaction.

\section{ROBOT SYSTEM FOR ORTHOPEDIC DIAGNOSIS - ROBOPED}

Knee-joint injuries due to work or sport-related accidents as well as degenerative illness are among the most common joint illness in the population (prevalence $20 \%$ ). A successful therapy and efficient rehabilitation depends very strongly on the right diagnosis. An accurate assessment of the mechanical abilities of the sick joint is highly important for its rehabilitation. This has been hindered by the fact that up to now only non-standardized or subjective methods have been available to the treating Orthopedist. Misdiagnoses are, therefore, pre-programmed.

To make accurate and objective evaluations of kneejoint stability, a Robot System for Orthopedic Diagnosis (ROBOPED) was developed at Fraunhofer IPK. The system can automatically carry out nearly all usual orthopedic tests on the knee (e.g., front/back drawer test, Pivot-Shift-Test, etc.). ROBOPED can also carry out complex, active and passive tests which combine knee movement and strain. The ROBOPEDSystem can also be used as a highly efficient rehabilitation device with three different programmable movements and strain loads (active/passive behavior, which can be generated with the help of force/impedance control).

The ROBOPED-System consists of a parallel Robot (Hexaglide-Structure - Figure 1). The Kinematics are laid out so that the entire spectrum of movement of the active and passive knee-joint as well as the movement radii of the lower leg are covered (Figure 2 ). In order for the parallel robot to follow the relatively large rotations of the knee joint during kneeflexion/extension, an additional passive rotating joint was built into the platform. The lower leg is fixed with the help of a platform, so that the spring joint is fixed and the movements of the knee-joint cannot be influenced. A 6D force/torque sensor is integrated into the platform. A gearless drive is employed as a driver with air bearings.

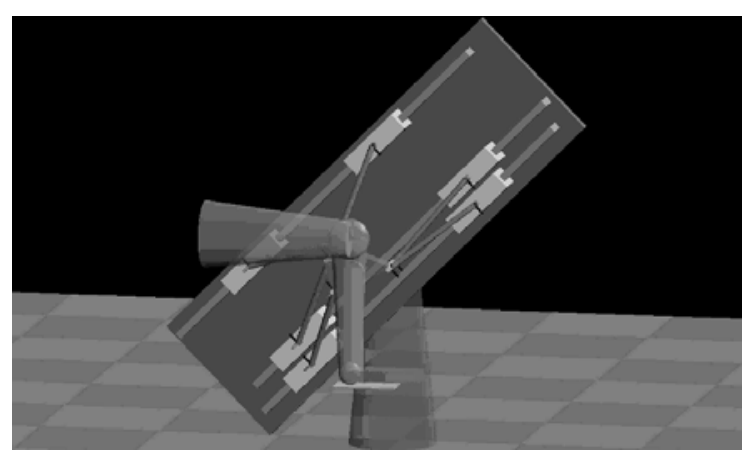

Fig. 1. ROBOPED-Kinematics Structure.

Hips and thighs are likewise fixed to the examination chair with an anatomical belt system. To compensate for small inaccuracies through small shifts in the skin (the so-called "skin effect") a special sensor was developed. With the help of a calibration procedure, the coordinate system of the knee of the patient is estimated. Via teaching, the robot "learns" what the individual anatomical flexion/extension movements of the patient. In doing so, the robot's force is regulated and the strength is set to zero. In addition, the impedance control is used as a basic method in order to move the knee and compensate for model uncertainties.

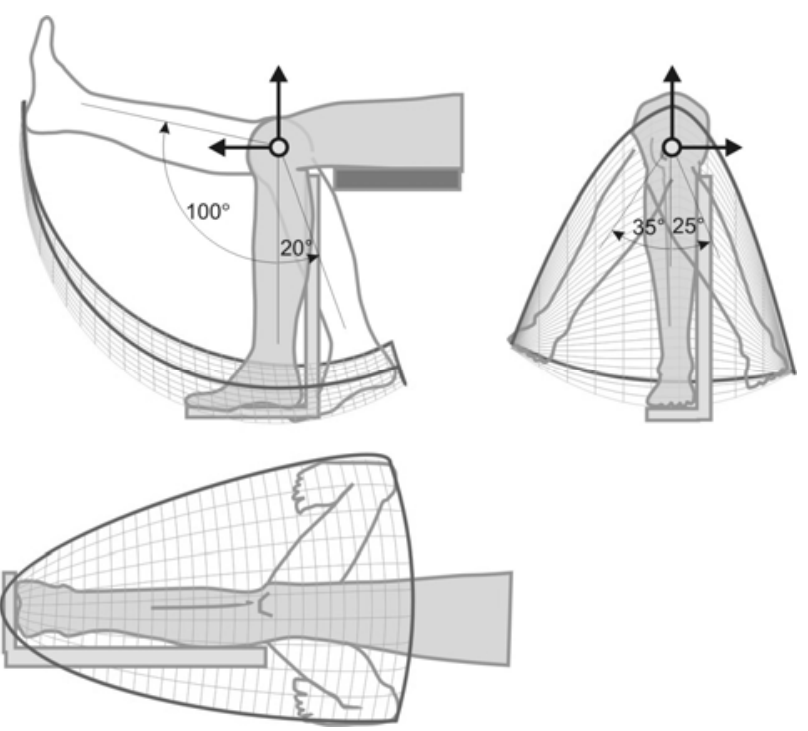

Fig. 2. Space of Movement of the ROBOPED. 
Figures 3 and 4 show the construction of the ROBOPED-Prototype, that is currently being prepared for clinical trials.

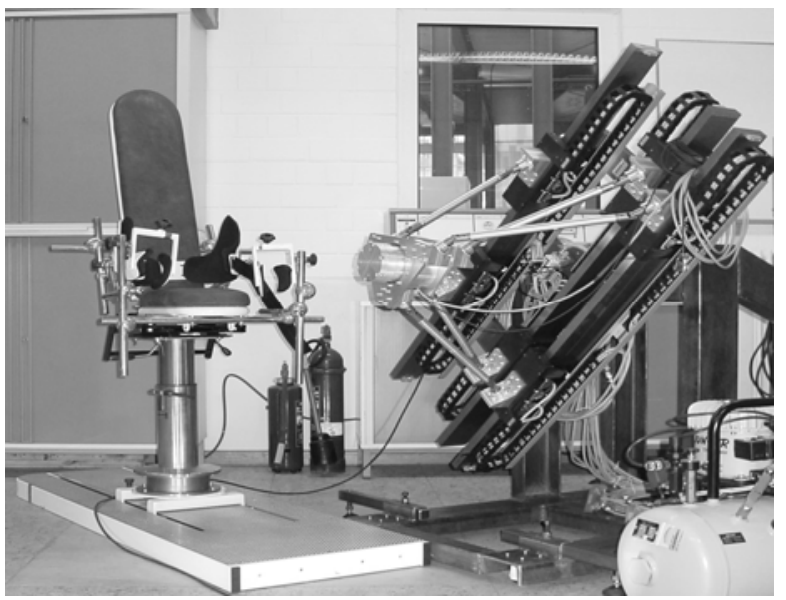

Fig. 3. ROBOPED-System.

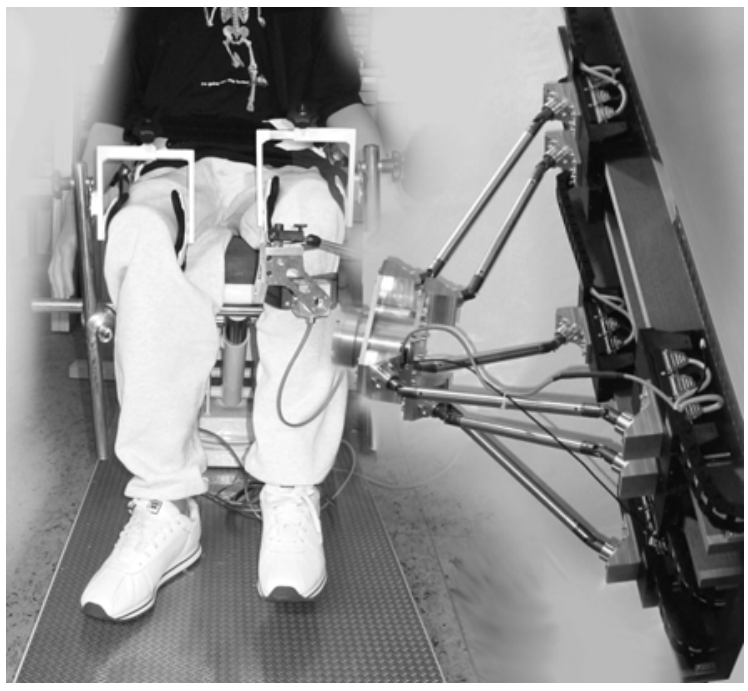

Fig. 4. ROBOPED, Patient-Robot-Interface.

\section{REHABILITATION ROBOT STRING-MAN}

The recovery or improvement of the ability to walk is a central concern of neurological and orthopedic rehabilitation (e.g., after a stroke, brain injury, paraplegia, and joint replacement). It takes highest priority for the patients, regardless of diagnosis, and is decisive for the reintegration of the patient into daily and working life. Modern concepts of motor learning require task-specific, repetitive exercise of as many walking situations as possible.

A further, important development at the Fraunhofer IPK Berlin in the area of interactive Human-RobotSystems, is the prototype of a robot system for motor rehabilitation, "STRING-MAN" (Figure 5). The goal was to develop a high-performance system for gait rehabilitation and restoration of motor functions for balancing. The starting point was the wish on the part of practicing doctors, that the upper body of their patients be able to be move in all 6 degrees of freedom and that weight be optimally balanced during locomotion therapy (the treadmill therapy with partial body-weight relief). For this, string kinematics with seven strings attached to a special corset was selected. This string robot is one of a kind. It is the first instance in which a human becomes integrated as part of the robot, as his body serves as the common platform. The movement of the strings is controlled via a linear Servo-drive. The whole system was designed with the help of an innovative optimization method for string kinematics (Surdilovic et al, 2004), which guarantees string tension for different body sizes and changing weight relief. Forces are minimized and distributed evenly across the upper body of the patient. Through this application, a force control compensates for the uncertainties of patient model, while at the same time supporting the movements initiated by the patient. Along with the position and force control, the control also allows various suspensions with regard to the spring and cushioning behavior as well as inertia (impedance control). For example, disruptive stimuli and movement restrictions in each direction and in each suspension variant could be clinically examined.

Beyond this, the patient can autonomously and safely test his ability to balance and stabilize himself using this unique system. Within the safety corset (around the patient's body) the control is done such that the string forces are minimized and the string tension and measurement of the position of the patient is made possible. In case of a fall or when the patient steps out of the safety area, his body is caught hold gently with the help of the impedance controlled string (Figure 5) and is brought again into the starting position. The methods used during therapy for the regulation of body balance were developed for the regulation of humanoid robots and were based on the so-called Zero-Moment-Point (Vukobratovic et al, 2002). Numerous sensors for force and position in the strings as well as sensors on the soles of the feet and a kneegoniometer support this control. The key element of the control is the control techniques for the synthesis of the impedance control (Surdilovic, 2003), which ensures the robust passivity or stability of the systems. These methods are used as the basis for the project's development.

The STRING-MAN-System is currently being clinically tested in cooperation with the Klinik Berlin. In a follow-up project (INNO-Reha) the introduction of the product is planned for next year. 

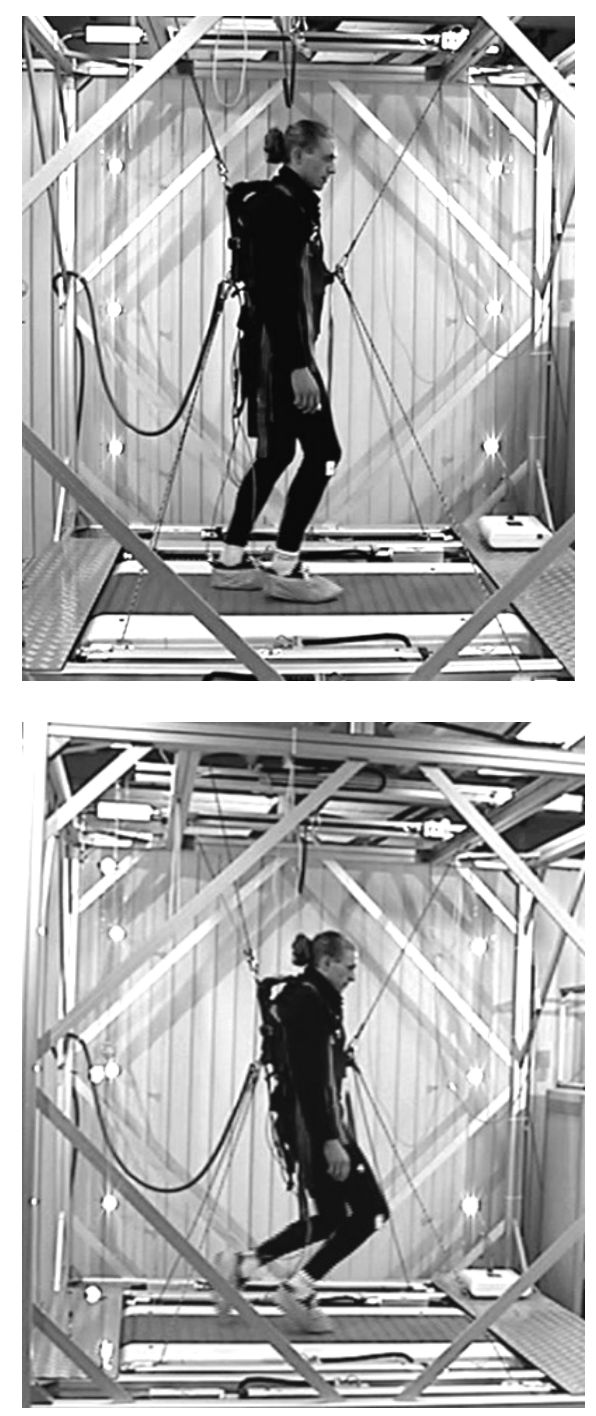

Fig. 5. Robot STRING-MAN: Robot with seven strings (above); Virtual safety corset for testing balance (below).

\section{COLLABORATIVE ROBOTS - KOBOTS}

The lifting, moving and manipulating of objects, that vary in size, form and weight are basic operation common to all branches of industry and enterprises. This kind of work is done in large measure by SME's. Although many technical instruments and devices have been developed over the last several decades, the repeated, one-sided handling of heavy, obstructive parts led to harsh physical demands on workers. Not uncommonly, these cause long-term health damage. According to a report of the European Agent for Safety and Health Protection, lifting and carrying of loads using bad posture are among the greatest risk factors for spinal injuries and impairments. The spread of work-related muscular and skeletal injuries has continued to increase despite this, and leads to a high degree of work stoppage. It has been shown statistically, that ca. $37 \%$ of workers in Germany suffer from lower-back disorders, approximately 100,000 are diagnosed as unable to work and go into early retirement, on a yearly basis. One third of these are related to muscle and skeletal injury. Thirty percent of all work-related accidents fall into these categories and cause production loss of 15 Billion Euros.

Over the last few decades, ever new technical devices and instruments have been developed in order to support handling processes. Systems which are currently on the market, such as hand-guided manipulators with balancing characteristics (e.g., manipulator arms, lifting units, hoists) already offer considerable improvements in the ergnomic characteristics of lifting and carrying. However, they still have far too many disadvantages, which continue to cause significant physical strain to the operator: Guidance and positioning depend exclusively on the skill of the operator; support from the system does not occur. The operator must overcome considerable inertial forces on his own. In order to remedy these

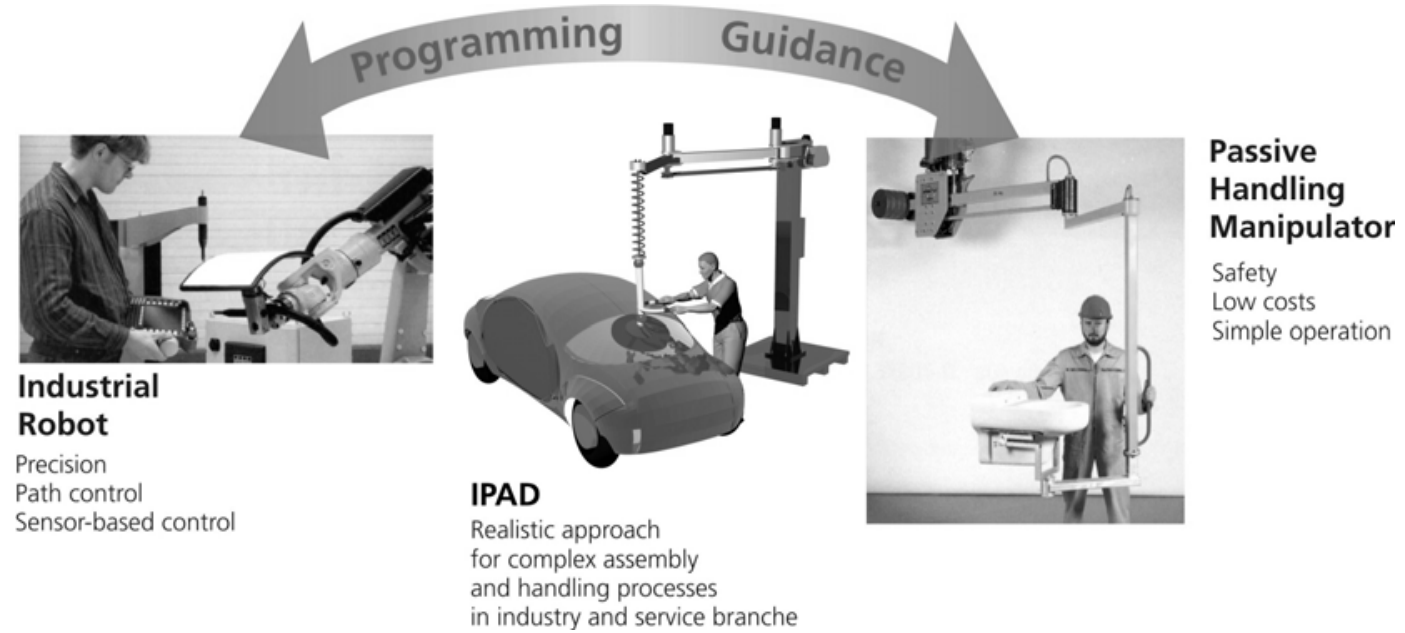

Fig. 6. Kobots, a new class of handling devices, which combine the characteristics of robots and hand-guided manipulators. 
disadvantages, new concepts for ergonomic and operator-friendly material-handling must be developed. Kobots offer a new, efficient (Figure 6) solution to this problem by offering real cooperation with the people. They are passive systems which are set in motion and guided by humans. They take over the ability to define and regulate a trajectory from classical robots, during the shifting of the end-effector (along so-called active barriers or virtual walls). The high prevision of the Kobots is comparable to that of a robot. In contrast to today's manipulators, they can compensate for the inertia of the device and the load without endangering human safety. Through these basic functions (guidance and force amplification), Kobots can reduce the physical strain put on humans to a minimum and thereby improve ergonomics and increase productivity and quality at the same time.

The first European prototype of a Kobot was developed at the Fraunhofer-Institut for Production Systems and Design Technology IPK (Figure 7). The functional principle employs a new CVT-System, utilizing modified differential gears. This allows the realization of optimal guidance with virtual walls, as well as force amplification (Surdilovic, 2003). The force amplification serves mainly to compensate process forces, for example, those of inertia during the operation of heavy parts, or the generation of clamping forces during assembly. Ergonomic operation and a reduction of the danger of injury are guaranteed in this manner. The Kobot should serve the human as a "third hand," helping instead of replacing him.

The IPK prototype should be further developed to for application in industrial systems (Figure 8). This new idea offers significant advantages with regard to costefficiency as well as control of force-amplification and path-guidance functions, which under gird Kobots. The further development will occur within the framework of the project. The core of this FuEproject is the integration of flexibility control in the control system of force amplifying manipulators. In this way, flexible walls will be programmed such that inaccuracies on the part of the operation can be compensated for, e.g., during positioning or montage.

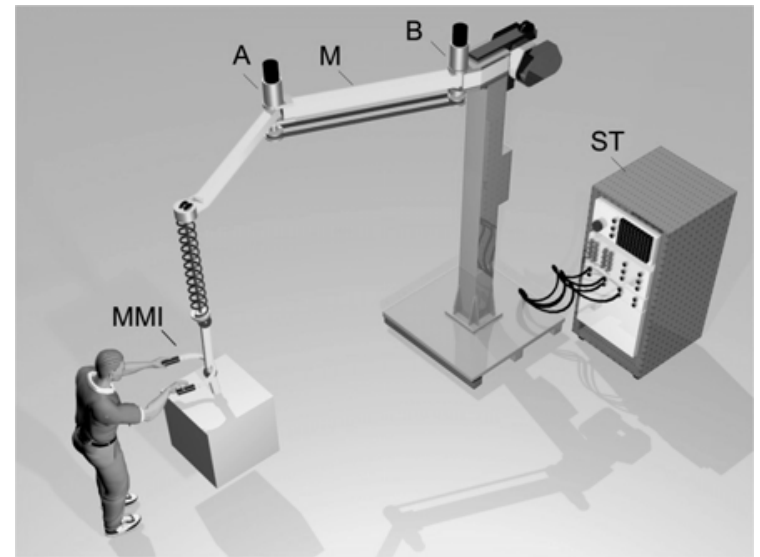

Fig. 7. Conceptual Construction of the IPK-KobotPrototype.

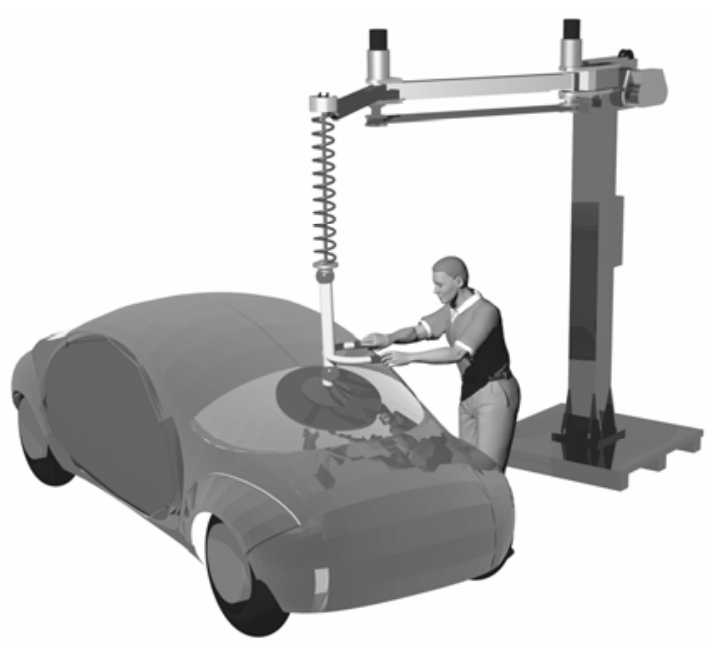

Fig. 8. Planned Industrial Application.

The programming of Kobots occurs by demonstrating the motion (Playback-Method), so that trained personnel is not necessary (Surdilovic, 2004) The first successful Tests with the new Kobots (Figure 9) were carried out by manipulator producers and industrial users, especially in the automobile industry, which has been followed with great interest. For this reason, Fraunhofer IPK together with cooperation partners, is planning to bring the first product to the market next year. 


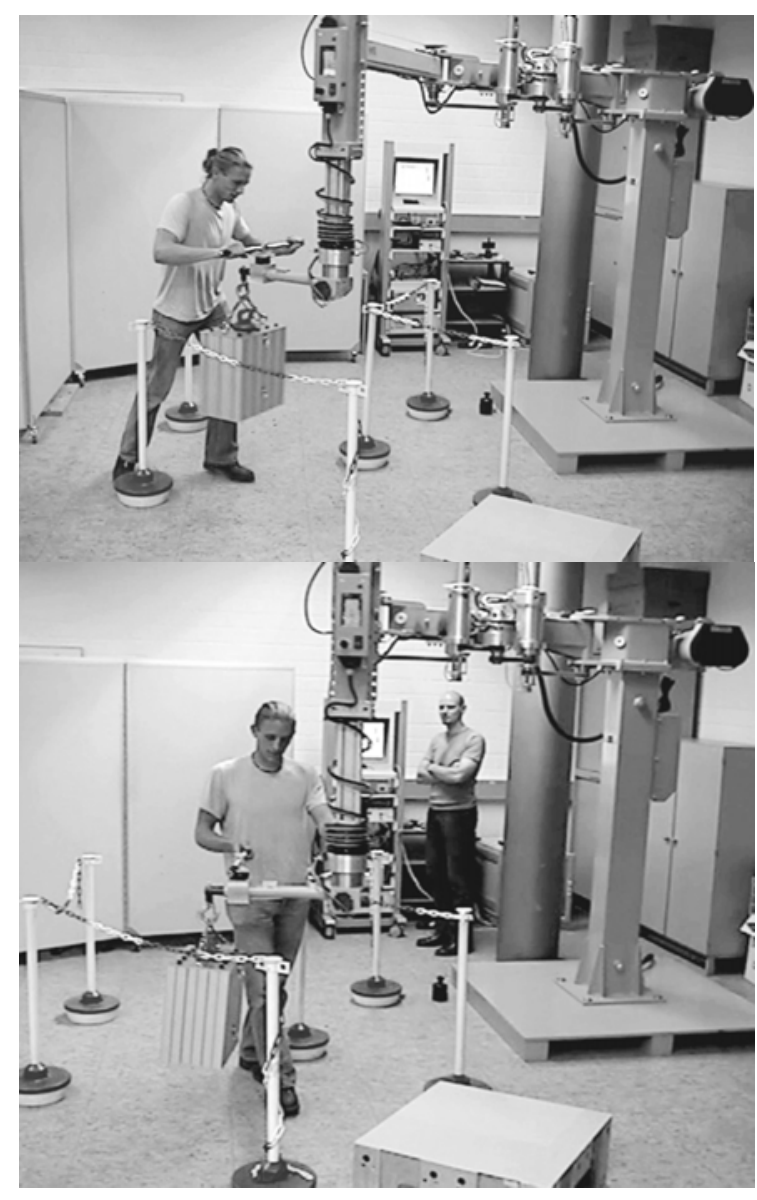

Fig. 9. New IPK-Kobot; above: traditional manipulator-operating mode, difficult maneuvering during movement of the cumbersome component between obstacles; below: Kobot operating mode, easy guidance obstacles. along a programmed track between

\section{CONCLUSION}

This contribution shows the newest developments at Fraunhofer IPK Berlin in the area of interactive $\mathrm{Hu}$ man-Robot-Systems for application in medicine (knee-joint diagnosis and gait rehabilitation) as well as in industry (material handling). Each of these systems allows the direct contact with humans; enable work with humans and interaction with humans (transfer of force and movement). Force sensors and special techniques for flexibility control are integrated into these advanced robot systems. The new prototypes, which have already shown their usefulness in laboratory experiments, will be clinically tested further developed within the framework of various clinical trials and industrial prototypes.

\section{REFERENCES}

Colgate J. E., Hogan N. (1992). Stability Problems in Contact Tasks. In: The Robotics Review 1 (ed. Khatib, Craig and Lozano-Perez), MIT Press, Cambridge, Massachusetts.

Šurdilović D., Bernhardt R. , Schmidt T., and Zhang J. (2004). STRING-MAN: A Novel Wire Robot for Gait Rehabilitation. Chapter 26 in: Advances in Rehabilitation Robotics- Human-friendly Technologies on Movement Assistance and Restora tion for People with Disabilities_(Ed. Zen Z.,

Stefanov D.), Lecture Notes in Control and Information Sciences, Vol. 306, Springer Verlag,.

Šurdilović D., Radojićić J. (2003). Robust Control of Human-Robot Interaction: Application for Motoric Rehabilitation. In: Proceedings Int. Conference on Rehabilitation Robotics ICORR'2003, Daejeon, Korea, pp. 112-116.

Šurdilovic D., Bernhardt, R., Zhang, L. (2003). New intelligent power-assist systems based on differential transmission. Robotica, 21, pp.295-302.

Šurdilovic D. (2004). Singularity Avoidance and Control of New Cobotic Systems with Differential CVT. In: Proceedings International Conference on Robotics and Automation, ICRA'04, New-Orleans, pp. 715-720.

Vukobratović M., Šurdilović D. (2002). Control of Robotic Systems in Contact Tasks. Chapter 21 in: The Mechanical Systems Design Handbook: Modeling, Measurement and Control _(Ed. Nwokah O.I. and Hurmuzlu Y.), CRC Press, Boca Raton. Vukobratović M., Borovac B, Surdilović D., Stokić D. (2002). Humanoid Robots. Chapter 27 in :The Mechanical Systems Design Handbook: Modeling, Measure ment and Control (Ed. Nwokah O.I. and Hurmuzlu Y.), CRC Press, Boca Raton.

Yanco H.A., Drury J.L. (2002). A Taxonomy for Human-Robot Interaction. In: Proceedings of the AAAI Fall Symposium on HumanRobot Interaction, AAAI technical Reports FS-02-03, Falmouth, Massachusetts, pp. 111-119.

Yanco H.A., Drury J. (2004). Classifying HumanRobot Interaction: An Updated Taxonomy. In: Proceedings of the IEEE Conference on System, man and Cybernetics. 